\title{
The Effects of Intermittent Energy Restriction on Indices of Cardiometabolic Health
}

\author{
Rona Antoni ${ }^{1}$, Kelly L. Johnston ${ }^{2}$, Adam L. Collins $^{1}$ and M. Denise Robertson ${ }^{1}$ \\ ${ }^{1}$ Nutrition, Metabolism and Diabetes Research Group, Faculty of Health and Medical Sciences, \\ University of Surrey, Guildford, UK
}

${ }^{2}$ Lighterlife UK Ltd, Cavendish House, Parkway, Harlow Business Park, Essex, UK

Correspondence should be addressed to: M. Denise Robertson; m.robertson@surrey.ac.uk

Received Date: 26 March 2014; Accepted Date: 6 May 2014; Published Date: 28 June 2014

Academic Editor: Maarten R Soeters

Copyright (C 2014 Rona Antoni, Kelly L. Johnston, Adam L. Collins and M. Denise Robertson. Distributed under Creative Commons CC-BY 3.0

\begin{abstract}
Intermittent energy restriction (IER) has become the subject of considerable scientific interest as a potential dietary approach for weight-loss and improving cardiometabolic health. This approach involves intermittent periods of total or partial energy restriction (ER) alternated with non-restricted intake, and has been studied in rodent and human populations.

This review aims to provide an overview of the IER literature to date, with a specific focus on its effects on cardiometabolic health indices in rodents and humans.

Current evidence from studies in rodents and humans suggests that IER is capable of promoting weight-loss and/or favourably influencing an array of cardiometabolic health indices, with equal or greater efficacy than conventional continuous ER approaches. Putative mechanisms include the effects of IER on adipose tissue physiology, stress resistance and fat distribution within visceral and intra-hepatic sites. However, a large proportion of this mechanistic evidence is limited to indirect observations and/or has come from rodent studies requiring translation into humans. Furthermore, whilst there is some indication that total IER and the array of partial IER protocols which have been developed may elicit distinct biological effects, our knowledge around this is limited as only a small number of rodent studies have directly addressed this.
\end{abstract}

Ultimately, whilst much remains to be learned about IER, including its mechanisms of action and long-term efficacy, the positive findings to date serve to highlight promising avenues for future research.

Keywords: "Intermittent Energy Restriction", "Intermittent Fasting", "Weight-Loss", "Cardiometabolic Health"

Cite this Article as: Rona Antoni, Kelly L. Johnston, Adam L. Collins and M. Denise Robertson (2014), "The Effects of Intermittent Energy Restriction on Indices of Cardiometabolic Health," Research in Endocrinology, Vol. 2014 (2014), Article ID 459119, DOI: 10.5171/2014.459119 


\section{Introduction}

Both the developed and developing World are currently in the midst of an obesity epidemic with its prevalence almost doubling in the last twenty five years. ${ }^{1}$ Whilst obesity rates are beginning to plateau, absolute levels remain high. In the UK, approximately $62 \%$ of adults were classified as overweight in 2012, and of these, 25\% were obese. ${ }^{2}$ Furthermore, national trends mask the worrying growth of morbid obesity, which almost tripled from $0.9 \%$ in 1995 to $2.4 \%$ in $2012 .{ }^{2}$ The development of obesity is considered to be a key contributor to an individual's cardiometabolic risk, i.e. risk of cardiovascular disease (CVD) and Type 2 diabetes (T2DM) ${ }^{3}$ which account for nearly $35 \%$ of deaths in the UK. ${ }^{4}$

A sustained modest weight-loss of 5 to $10 \%$ body-weight is associated with improvements in various indices of cardiometabolic health including but not limited to insulin sensitivity, vascular function, blood pressure and lipid levels. ${ }^{3}$ These beneficial effects of weight-loss can be partially explained by accompanying reductions in adipose tissue mass and dysfunction. 5 There is however, some evidence to suggest that energy restriction (ER) alone can confer metabolic changes which are independent to those of weightloss, by facilitating an acute negative energy balance 6-9 and/or by activating adaptive stress response pathways. ${ }^{10}$

The most commonly employed dietary approach to weight-loss involves varying degrees of continuous (daily) energy restriction (CER). However, when confronted with an obesogenic environment favouring sedentary lifestyles and passive overeating, successful weightloss and the necessary cognitive eatingrestraint required for CER becomes notoriously difficult to both achieve and maintain. Accordingly, only $20 \%$ of dieters are thought to be able to successfully sustain a $\geq 10 \%$ weight-loss for more than 12 months. ${ }^{11}$

In recent years, intermittent energy restriction (IER) has become the subject of considerable scientific interest as an alternative approach to weight-loss and/or improving metabolic health, and has been studied in rodent and human populations. Most rodent trials ${ }^{12-30}$ and preliminary trials in non-obese humans ${ }^{31-34}$ have used total IER protocols in which energy intake is completely restricted on alternate days. However, debate regarding the maintainability of such an approach longterm led the authors of one of these first-inman trials to propose that the addition of one small meal on a "fasting" day may improve the long-term acceptability of IER. ${ }^{33}$

Subsequent to this, weight-loss trials within human populations $35-52$ as well as some rodent trials $22,27,53,54$ have used an array of IER protocols which permit a small energy load to be consumed on the "fast" day, so that energy intake is partially but not completely restricted. The number of ER-days per week and degree of ER of these partial IER protocols has varied between studies, but ultimately this approach entails intermittent periods of very low energy intake, ranging between $15-50 \%$ of energy requirements. Individuals are then asked to consume an ad libitum or euenergetic intake on nonrestricted days.

For some, the IER approach may promote greater compliance than daily CER as an individual need not restrict their intake every day, and thus may potentially facilitate more sustainable weight-loss and maintenance. Furthermore by repeatedly inducing acute periods of negative energybalance and intermittent stress, the putative benefits of IER may extend beyond that of weight-loss alone.

The purpose of this review is to provide an overview of the IER literature to date, with a specific focus on its effects on cardiometabolic health indices in rodent and human populations. Potential mechanistic differences between IER and conventional CER approaches will be discussed, as will the implications for future research. 


\section{Intermittent Energy Restriction and Type 2 Diabetes Risk}

Rodent Models: Interventional IER trials in animal populations have exclusively used rodent models. Compared to ad libitum fed controls, rodents maintained on IER $(100 \%$ ER/alternate days) for periods of 12 to 24 weeks typically exhibit lower posttreatment levels of fasting glucose 14, 16, 17,29 and insulin. 16, 17,29 Using this particular IER protocol, Jiang et $\mathrm{al}^{28}$ have also demonstrated an increase in oral glucose tolerance and tissue insulin sensitivity (measured using a hyperinsulinemiceuglycemic clamp) after four weeks in healthy rats.

In one direct comparison of IER $(100 \%$ ER/alternate days) and CER (40\% ER/day), fasting glucose and insulin levels of mice in both groups were equally significantly lower than ad libitum fed controls following $20 \quad$ weeks. $^{15}$ Perhaps unexpectedly, in the IER group, this effect was noted in the absence of an overall reduction in either total energy-intake or adiposity suggesting that the IER-fed mice were not in fact in an energy deficit.

One study has tested the efficacy of IER (100\% ER/alternate days) in preventing the development of high-fat diet-induced insulin resistance (IR). ${ }^{54}$ In this study, rats received ad libitum access to either standard (control) or high-fat chow. At four weeks, rats in the high-fat group were then separated into two groups: one half continued to receive daily access, whilst the other half were fed on alternate days (i.e. underwent IER) for a further six weeks. IER resulted in significant reductions in bodyweight and intra-abdominal adipose tissue, which by 10 weeks were comparable to the control-fed animals. Despite this, skeletal muscle glucose uptake and insulin tolerance were impaired equally in both high-fat groups. Biopsies revealed significant declines in the expression of glucose transporter 4 (GLUT-4) within the skeletal muscle of both daily $(-30 \%)$ and IER $(-42 \%)$ high-fat groups relative to controls. This presents a possible mechanism for these observations, owed to the close relationship between skeletal muscle GLUT-4 content and maximal insulin-stimulated glucose transport capacity. 55

Humans: Preliminary Trials Using the Alternate Day Total Fasting Approach: To date, four trials in non-obese (i.e. healthy or overweight), glucose tolerant humans have assessed the effects of IER $(100 \%$ ER/alternate days) on glucose regulation. ${ }^{31-34}$ In one non-controlled trial by Halberg et $\mathrm{al}^{31}$, significant improvements in whole-body glucose uptake, assessed using a hyperinsulinaemic-euglycaemic clamp, were observed in men after as little as two weeks of IER in the absence of changes in body-weight. Conversely, Soeters et $\mathrm{al}^{34}$, who used a balanced cross-over study design, failed to note any effect of IER on whole-body glucose disposal after two weeks in men, when compared to two weeks on a standard euenergetic diet. No changes in fasting levels of insulin and glucose have been found by IER trials which have lasted between two to three weeks. ${ }^{31,33,34}$

A further non-controlled trial, which studied the effects of IER on glucose and insulin responses to a mixed test meal, highlighted the potential for gender disparities in postprandial glucose tolerance. $^{32}$ After three weeks, significant reductions in postprandial insulin responses were observed in male subjects (but no effect on glucose disposal), which is in contrast to the deterioration in the metabolic clearance of glucose noted only amongst female subjects. The lack of effect at the glucose disposal level amongst males in this study may be explained by their tendency for greater baseline glucose disposal rates, as has been observed by others. ${ }^{56}$

Additionally, on closer examination of the study design, post-treatment oral glucose tolerance was assessed following a totalfast day (i.e. after 36 hours of fasting) whereas baseline glucose tolerance was assessed after a standard 12-hour overnight fast. Fasting-associated metabolic alterations could be responsible for these apparent impairments in glucose 
tolerance (relative to baseline). These include elevations in non-esterified fatty acid (NEFA) mobilization (typically greater in women) and the secretion of counterregulatory hormones, which act to oppose insulin action and impair glucose disposal. ${ }^{57}$ Previous works have noted similar deteriorations in glucose tolerance in response to a mixed test meal 58, intravenous and oral glucose loads ${ }^{59}$, albeit following longer fasting intervals of 72 to 96 hours.

Given the potential long-term health implication of undergoing repeated cycles of exaggerated postprandial glucose responses, for example on the cardiovascular system ${ }^{60}$, further research is clearly warranted. Furthermore, to evaluate the true chronic treatment effect, future trials should ensure standardised periods of fasting are used before performing clinical testing, and that they incorporate a suitable control group.

Humans: Trials Using the Intermittent
Very Low Energy Approach: Studies which have used IER protocols that permit a small energy load to be consumed on restricted days are discussed next, with findings from randomized-controlled trials (RCTs) summarized in Table 1 . Within diabetic populations, hyperglycaemia is considered the hallmark diagnostic marker of metabolic abnormality and a major contributor to T2DM associated macroand micro- vascular complications. ${ }^{61}$ One study by Ash et al $^{36}$ saw 51 overweight/obese male subjects with T2DM assigned to one of three groups; (i) IER (four days $50 \%$ ER, three days ad libitum intake/week), (ii) CER (30\% ER/day, all meals provided) and (iii) CER (30\% ER/day, food self-selected by the participant). After 12 weeks, all groups exhibited comparable reductions in energy intake, anthropometrical measurements and glycated haemoglobin $\left(\mathrm{HbA}_{1} \mathrm{c}\right)$, a long term marker of glycaemic control. Conversely, within non-diabetic populations, periods of IER (75-85\% ER on restricted days) do not typically affect fasting glucose levels $37,41,45,48$ or $\mathrm{HbA}_{1 \mathrm{c}}{ }^{41}$, ${ }^{48}$; results of which can often be replicated by short term CER studies. ${ }^{62-65}$ These findings are unsurprising given that frank hyperglycaemia within the T2DM diagnostic range is effectively a late-stage manifestation of IR, which along with compensatory increases insulin secretion, can precede the onset of T2DM by many years. ${ }^{66,67}$ Findings from one large scale prospective cohort study, Whitehall II, reveal a sharp increase in the trajectory towards fasting hyperglycaemia which is only detectable three years prior to diagnosis with T2DM. ${ }^{67}$ Consequently, it can be argued that changes in circulating insulin concentrations, fasting (hepatic) insulin sensitivity and glucose uptake/clearance are more sensitive markers of deteriorating glucose control than fasting glycaemia in non-diabetics. ${ }^{68-70}$

In non-diabetic populations, three RCTs (Table 1) have used homeostasis model assessment (HOMA), a validated tool which can be used as an approximation of fasting hepatic insulin sensitivity. ${ }^{71}$ One trial found no effect of IER (75\% ER/alternate days) on fasting insulin sensitivity in overweight/obese men and women, either post-treatment or relative to ad libitum controls. ${ }^{46}$ Conversely, two studies in overweight/obese women, which have used IER protocols designed to deliver an average weekly energy deficit of $\sim 25 \%$ with two consecutive $75 \%$ ER days per week, have demonstrated post-treatment improvements in both fasting insulin and insulin sensitivity which have exceeded that of energy-matched CER (25\% ER/day) controls after three months 48 and six months. ${ }^{41}$ 


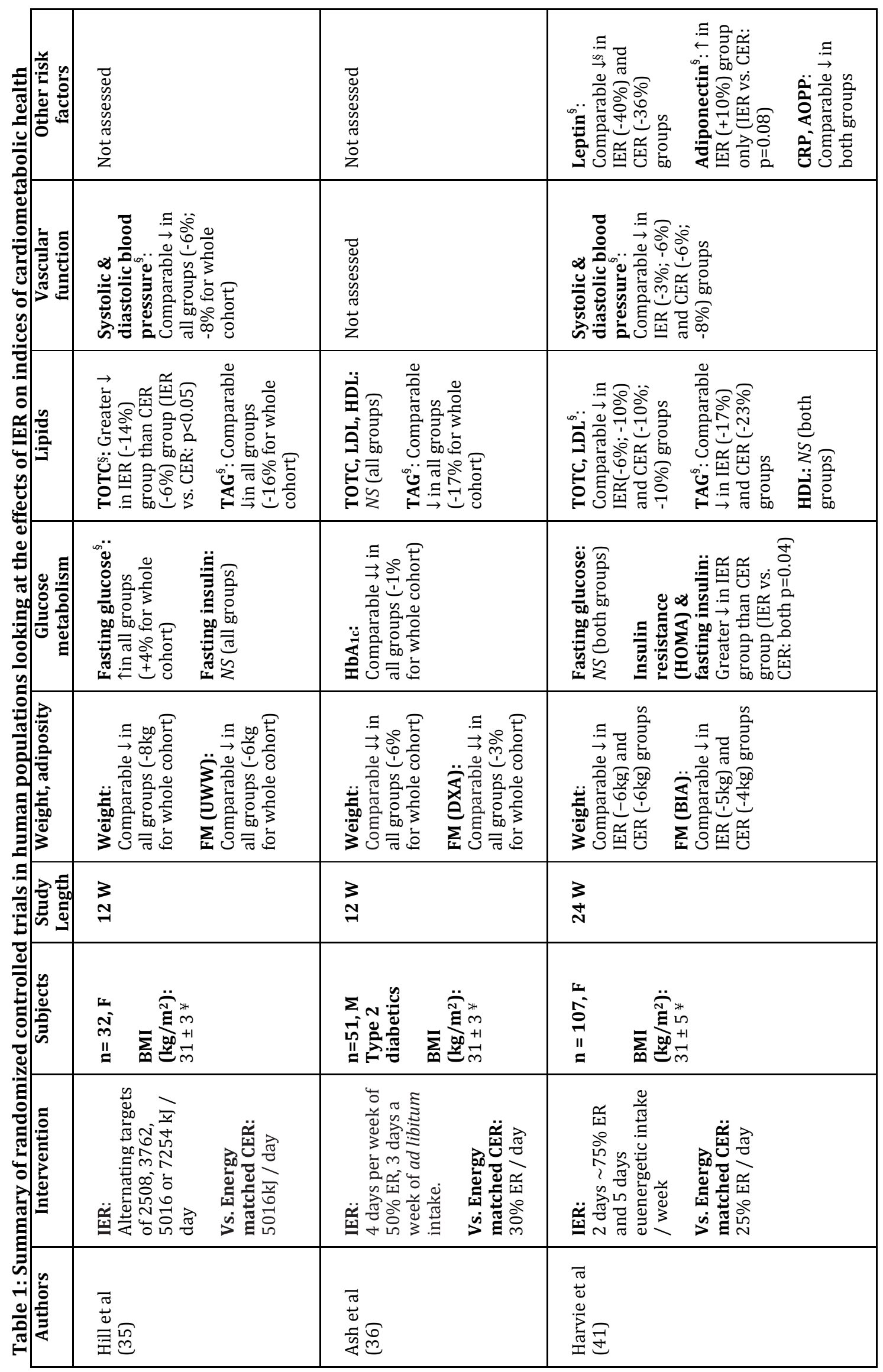




\begin{tabular}{|c|c|c|}
\hline | & 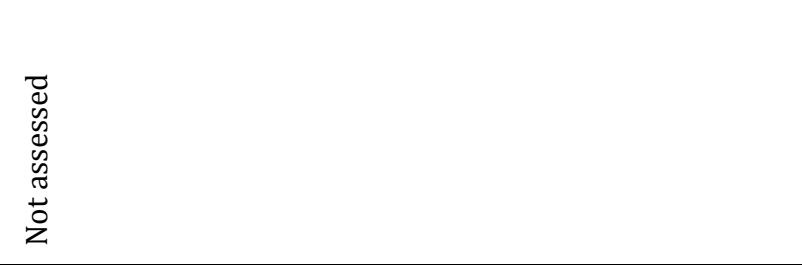 & 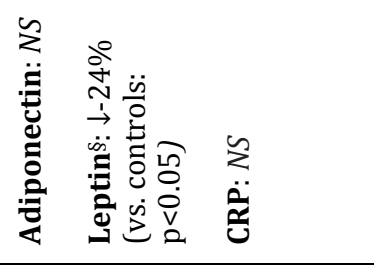 \\
\hline 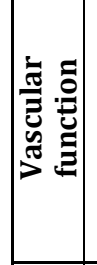 & 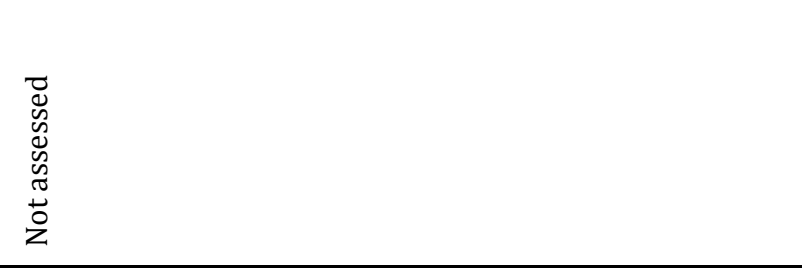 & 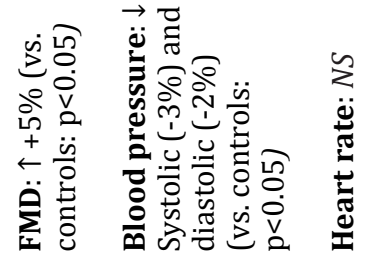 \\
\hline$\frac{n}{3}$ & 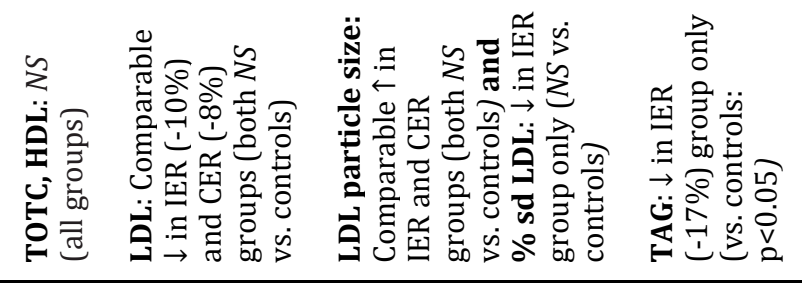 & 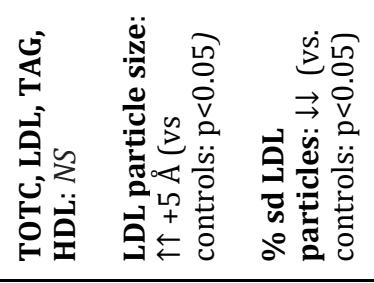 \\
\hline 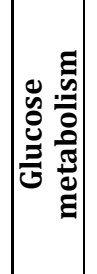 & 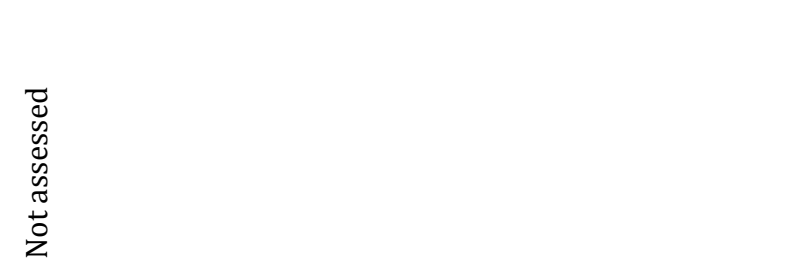 & 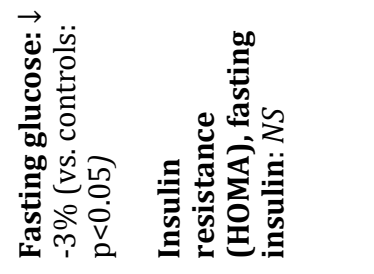 \\
\hline 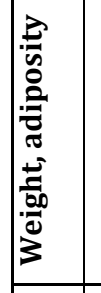 & 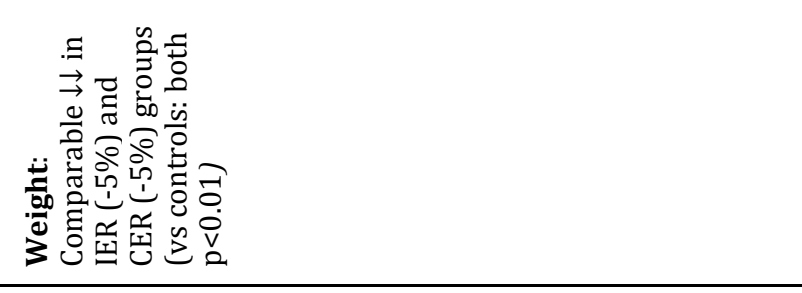 & 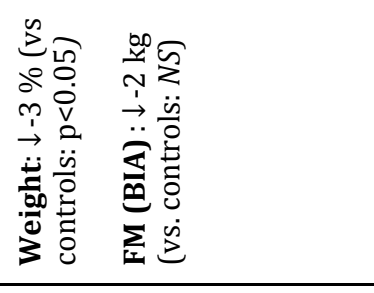 \\
\hline 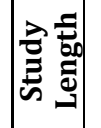 & $\begin{array}{l}3 \\
\underset{N}{3}\end{array}$ & $\begin{array}{l}3 \\
\mathbb{N}\end{array}$ \\
\hline 峞 & 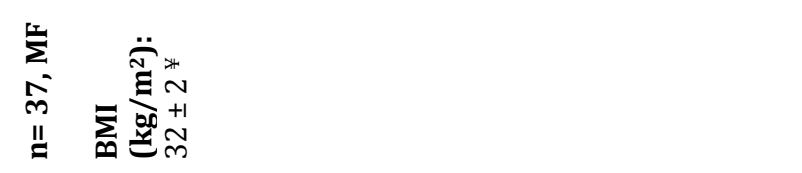 & 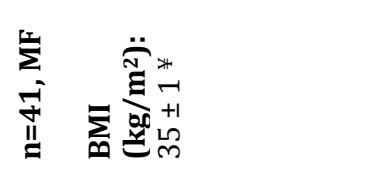 \\
\hline 递 & 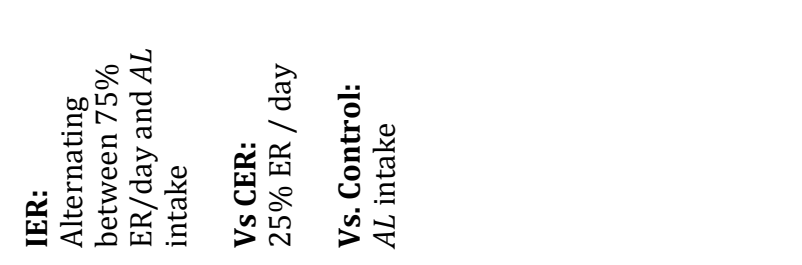 & 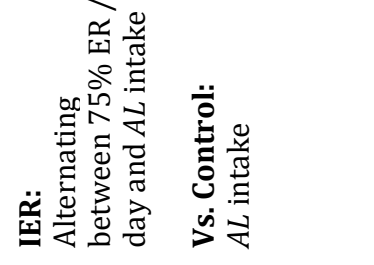 \\
\hline 管 & 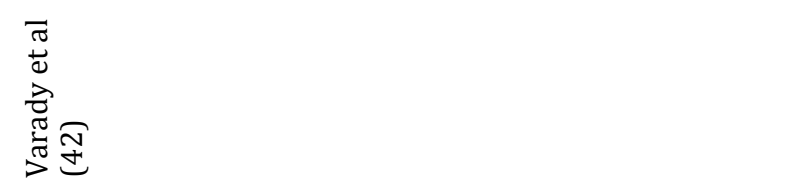 & 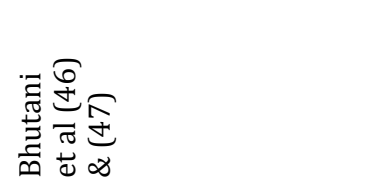 \\
\hline
\end{tabular}




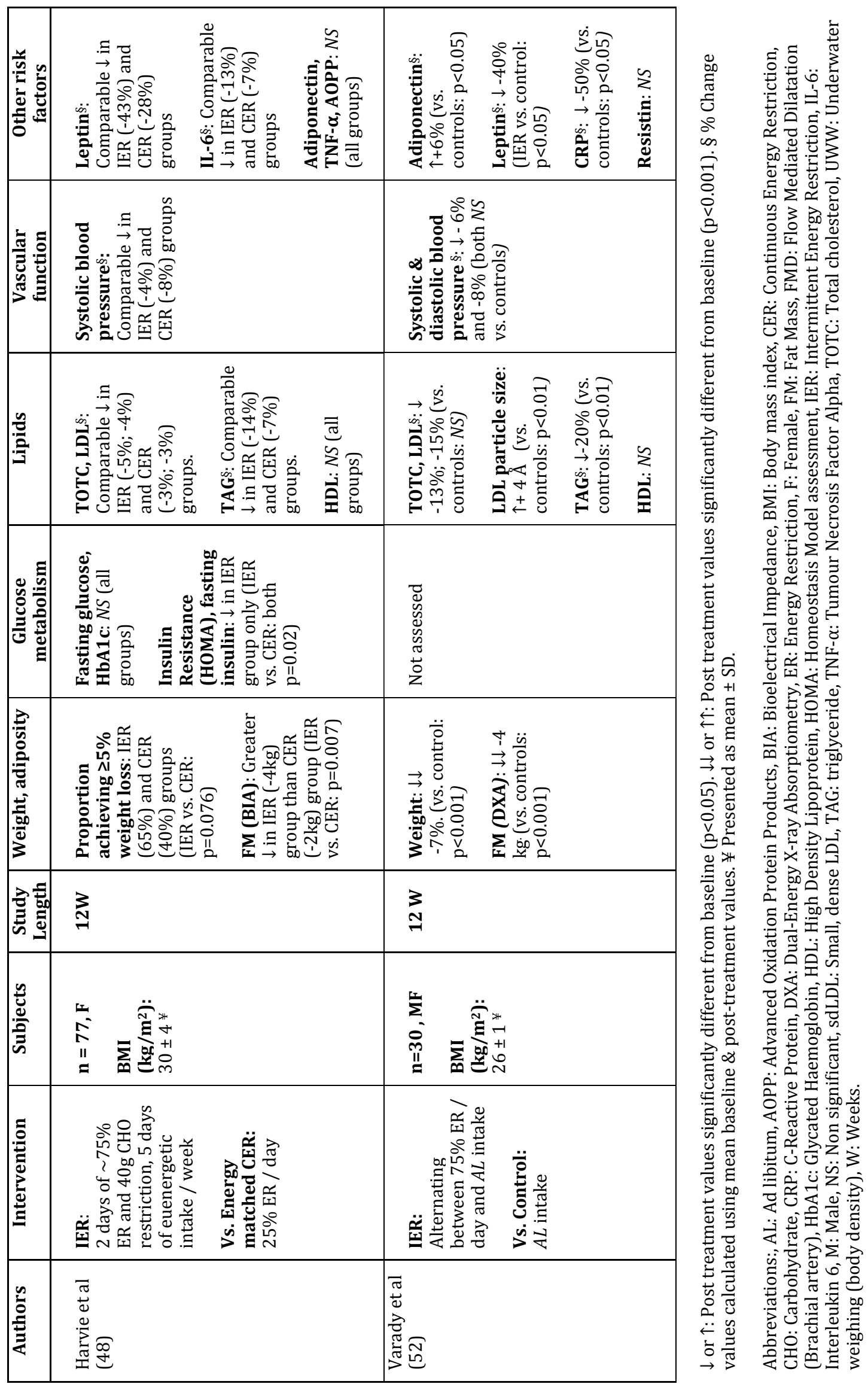




\section{Intermittent Energy Restriction and Cardiovascular Health}

Rodent Models: A growing number of trials have examined the effects of IER (100\% ER/alternate days) on cardiovascular risk factors in rodent models. Of these, several have reported post-treatment reductions in total cholesterol 12,53, triglyceride 12,53 and increases in high density lipoprotein (HDL) cholesterol. $^{20}$ In studies using normotensive rats, three to six months of IER has been observed to lower blood pressure $16-19$ and heart rate $16,17,19$, with the magnitude of the change comparable to CER (40\% ER/day) rats. ${ }^{19}$ In accordance with these findings are improvements in aortic endothelium-dependent and heart rate variability (a marker of sympathovagal balance) in IER-fed rats. ${ }^{72}$

Chronic IER appears to attenuate the agerelated decline in both cardiac diastolic function $^{30}$ and fibrosis ${ }^{24}$ after 24 months. Several trials have demonstrated that the cardiomyocytes of rodents maintained on IER become more resilient to ischaemic injury induced by occlusion of the left coronary artery 18, 21, 29, which translated into improved long-term survival following such injury in one study. ${ }^{21}$ In addition, within these studies, associations have been found between the cardiovascular improvements and markers of oxidative stress 24,30 , inflammatory responses $18,24,29$, 30 and increases in circulating levels of adiponectin. ${ }^{29}$ Collectively, these reported changes in biochemical and physiological cardiovascular risk factors would be expected to suppress atherosclerotic development and preserve cardiovascular health.

\footnotetext{
Humans: Preliminary Trials Using the Alternate Day Total Fasting Approach: In non-obese humans, short-term two to three week intervention periods of IER (total fast/alternate days) do not appear to influence resting heart rate 31 or blood pressure. ${ }^{33}$ One three-week IER study noted gender specific treatment-effects, which may in part relate to baseline differences and simple regression to the mean. In this study, post-treatment elevations in HDL cholesterol levels were
}

only noted in female subjects. On the other hand, triglyceride levels were solely reduced in male subjects who, at baseline, possessed higher fasting levels than their female counter-parts. ${ }^{33}$

Humans: Trials Using the Intermittent Very Low Energy Approach: In overweight and/or obese populations, the majority of IER (60-85\% ER/alternate days) trials spanning four to 12 weeks have consistently demonstrated global improvements in cardiovascular risk markers, encompassing enhancements in glucose metabolism (described above) and lipid profiles $37-40,42,46,49,50-52$ relative to baseline, which collectively would be expected to improve vascular function. Conversely, the effects of IER on blood pressure, which have been studied within predominantly normo- to pre-hypertensive cohorts, have been inconsistent, with some $38,40,46,45$ but not all studies 37,51 reporting decreases in systolic and diastolic blood pressure ranging from between - 3 to $-13 \%$ and -2 to $-13 \%$ respectively.

Whilst the association between elevated LDL cholesterol levels and cardiovascular disease are well established, lipoprotein particles exist upon a continuum, varying in terms of physiochemical properties and atherogenicity. Compared to larger, more buoyant LDL particles, small dense LDL are considered particularly atherogenic due to their enhanced ability to penetrate into the sub-endothelial space and increased susceptibility to oxidation. ${ }^{73-75}$ Evidence from several controlled (Table 1) and noncontrolled studies suggests that periods of IER (75\% ER/alternate days), in addition to reducing LDL levels, are also able to increase LDL particle size and induce favourable shifts in the distribution of LDL towards larger, LDL subfractions in overweight/obese subjects. ${ }^{42,} 46,51$ These findings have also been replicated by studies which have used combined fasting (one day/week) and moderate ER (20\% ER for remaining six days/week) protocols. ${ }^{44}$, 76

In contrast to LDL, the HDL cholesterol sub-fraction fulfils a vital role in maintaining vascular health through its 
anti-oxidative, anti-inflammatory, antithrombotic properties. It also transports cholesterol from extra-hepatic sites, including the arterial wall, to the liver for excretion via reverse cholesterol transport.77 Results from IER (60-85\% ER/alternate days) trials have thus far been inconsistent, with some reporting decreases ${ }^{40}$, and others increases 37,39 , but with the majority showing no effects on HDL cholesterol levels. $38,42,43,45,49,51$, ${ }^{52}$ Inconsistencies may have arisen due to the biphasic response of HDL documented by studies of ER induced weight-loss, whereby levels typically decrease during active weight-loss then either return to baseline or (less commonly) rise following attainment of weight stability.78 Complementary increases in HDL levels and particle size have been shown when endurance exercise have been combined with IER $42,46,47$ however further discussion goes beyond the scope of this review.

Two IER trials in overweight/obese men and women have looked at markers of endothelial function 47,49 , abnormalities of which can be detected long before overt cardiovascular disease develops. ${ }^{79} 12$ weeks of IER $(75 \%$ ER alternated with $125 \%$ intake) led to a $+5 \%$ increase in brachial artery flow mediated dilatation (FMD), indicative of improved vascular health ${ }^{47}$ (Table 1). Klempel et $\mathrm{al}^{49}$ also noted an increase in brachial artery FMD (of 2\%) after eight weeks of a similar IER protocol, but only in those consuming a low-fat background diet $25 \%$ of total energy intake as fat on non-restricted days). In contrast, subjects who consumed a high-fat diet ( $45 \%$ of total energy intake as fat on non-restricted days) exhibited a$2 \%$ decrease. The inability of IER to mitigate the negative effects of a high-fat diet upon markers of vascular function, despite comparable improvements in lipid profiles and weight-loss, suggests that individuals will still need to employ healthy eating principles on non-restricted days. It also highlights the need to exercise caution when assessing individual risk factors in isolation, and the value of markers such as FMD which represent the cumulative effects of various determinants of vascular function.

\section{Chronic Intermittent Energy Restriction and Possible Adverse Effects}

In contrast to the vast majority of studies are two rodent trials which highlight the potential for negative effects when IER $(100 \%$ ER/alternate days) is used chronically. One eight-month IER trial in obesity-prone rats reported an increase in the production of reactive oxygen species (ROS), with no clear mechanistic explanations. ${ }^{26}$ Another trial, this time over six months, showed that IER-fed rats displayed marked reductions in diastolic compliance and cardiac reserve due to observed reductions in cardiomyocyte size and increased myocardial fibrosis. ${ }^{23}$ No human trials to date have reported any adverse effects on cardiovascular health during long-term IER. Conversely, other acute studies in humans have yielded possible mechanistic insights. Specifically, similar impairments in ventricular diastolic function have been observed in healthy men following short-term (three-day) very low energy diets (VLEDs). ${ }^{80,81}$ Also noted by these studies were elevations in NEFA mobilization and a resultant increase in myocardial triglyceride content, which has been associated with ventricular dysfunction. Whilst not fully understood, complex mechanisms involving intermediates of NEFA metabolism and oxidative stress are believed to be responsible for this lipotoxic effect on the myocardium..$^{80,81}$

\section{Intermittent Energy Restriction: Acceptability and Effects on Feeding Behaviour}

Free-living humans are especially vulnerable to the effects of obesogenic environmental and behavioural pressures, which pose a significant barrier for the continued success of weight-loss attempts. This is further compounded by elevations in metabolic efficiency (whereby energy expenditure declines beyond that predicted from the change in metabolic mass) and appetite which accompany weight-loss, and may ultimately predispose to weight regain. ${ }^{82}$ Moreover, changes in neural activity within brain regions known to be involved in regulatory, emotional and cognitive 
control of food intake have also been observed following weight-loss. ${ }^{83}$

This poses the question of whether feelings of hunger and negative mood states, particularly during restriction periods could become self-limiting factors for the success of IER in the long-term as either a weight-loss or maintenance strategy. Persistent hunger and irritability noted in non-obese men and women in earlier IER trials, where food was completely restricted on alternate days, suggest that some individuals are unlikely to be able to comply for extended periods of time. ${ }^{33}$ Compliance with IER protocols which allow some intake on restricted days have been promisingly high. ${ }^{38,} 43,48,49, \quad 51,52$ Additionally, several studies have also demonstrated a lack of compensatory hyperphagia $38,39,41,48,52,84$ upon resumption of free-eating which, if were to occur, would be expected to limit the efficacy of IER as a weight loss/ maintenance strategy.

Subjective measures of hunger in these IER weight-loss trials have been shown either to remain stable 37,52 , or to decrease over the study interval. ${ }^{84}$ When interpreting these findings, it should be noted that assessment of appetite is highly variable between individuals. ${ }^{85}$ Additionally, overweight/obese subjects entering weight-loss trials are self-referring and so typically exhibit greater levels of motivation and cognitive eating-restraint. This may favourably influence their ability to resist hunger and hence bias subjective measures of appetite..$^{82}$ Furthermore, the effects of weight-loss upon subjective appetite measures are most frequently detected in pre-meal and post-absorptive states, indeed, the use of visual analogue scales have only been validated for this purpose. ${ }^{85}$

To date, the longest weight-loss trial of IER, lasting six months, demonstrated comparable weight loss amongst overweight / obese women assigned to IER (two $\sim 75 \%$ ER days per week) and CER (25\% ER/day) groups at all tested time points. ${ }^{41}$ Whilst this suggests that IER is no easier to follow in the long-term, the limited variety of foods permitted on restricted days in this particular study (milk, fruits and vegetables) may have limited the acceptability and long-term sustainability of this protocol. In a later trial lasting three months, also in overweight/obese women, a similar twoday IER protocol was used with the addition of a $<40 \mathrm{~g}$ ER-day carbohydrate restriction. Subjects were permitted to consume a greater variety of protein-rich lean meats, fish and eggs which may have facilitated greater acceptability and satiation. ${ }^{86}$ Consequently, compliance amongst IER subjects was high and may well have contributed to its observed superiority over CER in reducing fatmass. ${ }^{48}$

Increased sensations of appetite associated with weight-loss are thought to relate in part to alterations in the neural and endocrine responses to energy deficit, which occur in an attempt to restore fatmass by increasing energy intake. ${ }^{82,} 87$ However, little is known about the effects of IER on physiological regulators of feeding behaviour and appetite. Two hormonal regulators which have been studied are leptin and ghrelin, whose effects on energy balance are in a large part mediated by the hypothalamus. ${ }^{87}$ Leptin, a peptide hormone secreted by adipose tissue, acts to reduce appetite ${ }^{87}$ Circulating levels are proportional to adiposity and so decline during weight-loss, thus increasing appetite, however this reduction is believed to be disproportionately greater than the change in fat-mass. ${ }^{82}$ As expected, IER $(60-85 \%$ ER on restricted days) weight-loss interventions have all noted a decline in leptin levels 39, 41, 46-49, 52, comparable with energy-matched CER protocols after three months ${ }^{48}$ and six months. ${ }^{41}$

Conversely ghrelin, produced mainly by $\mathrm{P} / \mathrm{D} 1$ cells lining the fundus of the stomach, exerts appetite stimulating effects and so levels are typically at their peak pre-meal and decrease post-prandially. ${ }^{87}$ As weightloss is associated with increased fasting levels of ghrelin 88 and reduced suppression in response to a meal in obese subjects ${ }^{89}$, changes in circulating ghrelin 
levels have been assessed in several IER studies. One three-week IER (100\% ER/alternate days) study in non-obese men and women noted no changes in posttreatment levels of ghrelin when measured after both 12 and 36 hours of fasting. ${ }^{33}$ Similarly another study, this time in overweight/obese women, did not detect any changes in fasting ghrelin after six months in subjects assigned to either IER (two $\sim 75 \%$ ER days per week) or CER (25\% ER/day). ${ }^{41}$ Clearly, homeostatic control of food intake is under the influence of numerous peripheral mediators, of which ghrelin and leptin are only two. ${ }^{82}$ It would therefore be important for future research to incorporate a more definitive assessment of gastrointestinal physiology for example for the full effects of IER to be determined. Future works should also seek to identify and assess the effects of IER on hedonistic mechanisms in addition to merely homeostatic factors, in order to gain a better insight into the effects of IER on feeding behaviour.

\section{Potential Mechanistic Differences between IER and CER}

Studies in rodent models and humans show that IER may improve a variety of traditional cardiometabolic risk markers. A schematic representation summarizing these improvements, the effects on clinical outcomes and proposed mechanisms, is presented in Figure 1.

\section{Changes to Adipose Tissue Physiology:} Once considered an inert storage depot for excess energy, adipose tissue has been clearly identified as playing an influential role in cardiometabolic health. ${ }^{3}$ Adipose tissue functions not only as an important buffer for fatty acids ${ }^{90}$ but also as a highly active secretory organ, capable of influencing whole-body physiology through the production of an array of bioactive adipokines. 91 Normal functioning of adipose tissue is often (but not exclusively) impaired in obesity 91 and can be characterized by excessive hypertrophic adipocyte growth, elevated macrophage infiltration, hypoxia and dysregulated lipid storage. ${ }^{92}$
One theory proposes that once the storage capacity of subcutaneous adipose tissue (SAT) depots is exceeded under conditions of energy excess, either as a result of impaired expandability and/or excessive hypertrophic growth, fat deposition within visceral depots and non-adipose tissues including the liver, skeletal muscle and pancreas can ensue. ${ }^{93}$ This can subsequently lead to the development of systemic IR and a series of associated cardiometabolic disorders including dyslipidaemia, dysglycaemia, hyperinsulinaemia and hypertension. ${ }^{3}$ Expression of pro-inflammatory mediators including interleukins 1 (IL-1), 6 (IL-6), tumour necrosis factor alpha (TNF- $\alpha$ ) and resistin, are also increased which can further potentiate IR and promote atherosclerosis. Conversely, secretion of adiponectin, a potent insulin-sensitizing, anti-atherosclerotic mediator, is typically depressed. ${ }^{3}$ Thus, cardiometabolic risk may not be due to the excess accumulation of adipose tissue per se but rather as a consequence of its dysfunction.

Findings from rodent trials suggest that IER (50-100\% ER/alternate days) is capable of modulating adipose physiology, independently of changes to fat-mass. For example, one study which has compared three IER protocols (25\%; 50\% and $100 \%$ ER/alternate days) with ad libitum fed controls, found that at four weeks, both the subcutaneous and visceral adipocytes of male mice maintained on IER $(50 \%$ and $100 \%$ ER/alternate day) were some $35 \%$ to $50 \%$ smaller than those of controls. ${ }^{22}$ Body weight was reduced in the IER (100\% ER/alternate days) group, whilst adipose tissue mass remained unchanged after all interventions. Two conclusions were drawn by the authors of this study. Firstly, that IER (50\% ER/alternate days) was capable of altering adipose physiology even in the absence of an energy deficit. Secondly, given there was no overall reduction in adipose depot weight in IER (50\% or $100 \%$ ER/alternate days) fed mice, this reduction in adipocyte cell size must reflect an increase in adipocyte number (i.e. adipogenesis). 


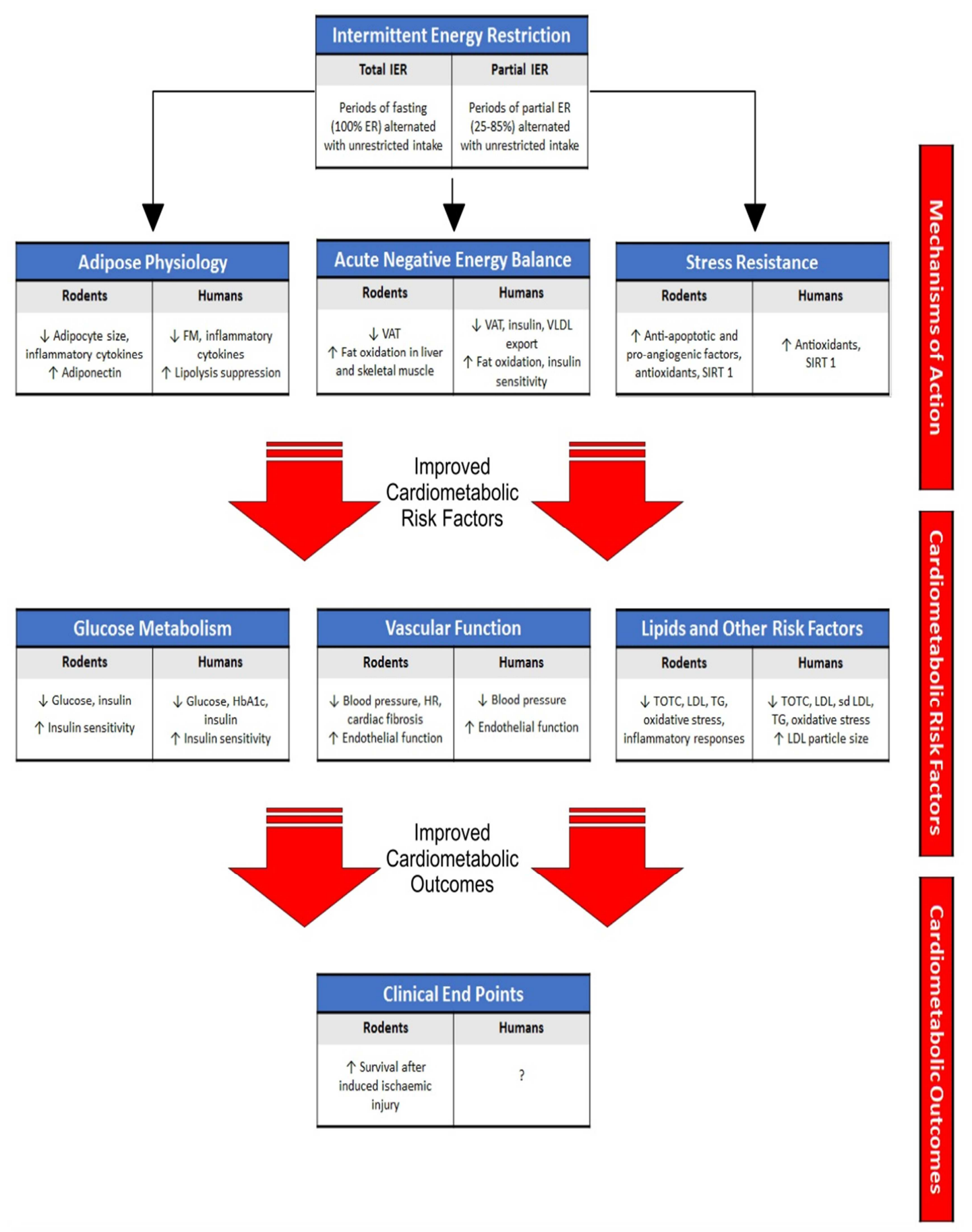

Figure 1: Intermittent Energy Restriction (IER) - Schematic representation of its mechanisms of action, effects upon cardiometabolic risk factors and clinical end points reported by animal and human IER studies to date. Potential mechanisms include the effects of IER upon adipose physiology, stress resistance and the metabolic benefits of acute negative energy balance. Rodent and human evidence suggest that these interact to improve various cardiometabolic risk factors including glycaemic control, lipid profiles, vascular function, oxidative stress and inflammatory responses. In rodents, this has also been shown to result in improved clinical end points including survival after induced ischaemic injury. Abbreviations: ER - Energy restriction, HbA1c: Glycated haemoglobin, HR: Heart rate, LDL - Low density lipoprotein, sd LDL: Small, dense LDL, SIRT1 - Sirtuin 1, TG - Triglycerides, TOTC - Total cholesterol, VAT Visceral adipose tissue, VLDL-Very low density lipoprotein. 
Weight-loss independent effects of IER have also been noted by one human trial, studying IER (100\% ER/alternate days) in non-obese men. ${ }^{31}$ In this trial, a reduction in adipose tissue lipolysis, which implies improved insulin sensitivity within adipose tissue, was observed despite no overall change in fat-mass after two weeks. After three weeks of IER however, a separate study noted a reduction in fat-mass, which suggests that unlike in the rodent studies mentioned previously, humans may be unable to fully compensate for energy deficits incurred on fast days with increasing durations. ${ }^{33}$

In overweight or obese subjects, IER (60$85 \% \mathrm{ER} /$ alternate days) have been shown to produce an average weight loss of between -5 and $-8 \%$ body-weight in studies of four to 12 weeks duration with the degree of weight-loss seemingly correlated to the length of the intervention. . $^{3}, 38,40,45-47$, 49-52 Weight-loss is accompanied by reductions in whole-body adiposity $37,38,40$, 45-47, 49-52, waist circumference $\mathrm{e}^{39,40,43,45}$ and favourable shifts in adipokine expression encompassing lowered circulating levels of TNF-a 37 , IL-6 37, resistin 39, 49 and augmented adiponectin expression 39, consistent with favourable modulations in adipose function. Also noted by one study was a decline in C-reactive protein (CRP) ${ }^{52}$, potentially the most widely accepted of the inflammatory markers and whose hepatic synthesis is induced by pro-inflammatory cytokines. ${ }^{94}$

Two RCTs in overweight/obese women have compared energy-matched IER and CER protocols (Table 1). In the longer of the studies, IER (two $\sim 75 \%$ ER days per week) demonstrated equal efficacy to CER (25\% ER/day) at reducing body-weight, adiposity and CRP after 6 months, whereas a post-treatment increase in adiponectin occurred only in the IER group. ${ }^{41}$ In followup work by the same group ${ }^{48}$, subjects were assigned a similar two-day IER protocol with the addition of a $<40 \mathrm{~g}$ ER-day carbohydrate restriction. Compared to CER (25\% ER/day), proportionally more IER subjects tended to attain a $>5 \%$ weight-loss after three months, whereas body-fat losses in the IER group were significantly greater. Accordingly, IER was found to be superior to CER in terms of improving insulin sensitivity. Reductions in IL- 6 were comparable between the groups, whereas neither intervention had an effect on adiponectin or TNF- $\alpha$.

Change in Energy Balance and Fat Mobilization: Dramatic improvements in metabolic control have been observed in morbidly obese individuals undertaking VLEDs ${ }^{6-9}$ with even short periods of severe energy restriction significantly improving both hepatic 9 and whole-body 7 insulin sensitivity. It is of particular interest to note that these metabolic improvements appear to occur before that of significant weight-loss.6, 8, 9

A recent review by Taylor 8 elucidates the role of the large negative energy balance elicited by VLEDs, which is now postulated to be the primary mechanism behind these metabolic effects. According to the "Twin Cycle Hypothesis", chronic energy excess (in the presence of skeletal muscle IR) results in a compensatory hyperinsulinaemia in an attempt to maintain normoglycemia, however will also promote the accumulation of intrahepatic lipid (IHCL) via the stimulation of de novo lipogenesis. Hepatic IR ensues, increasing endogenous glucose production, hence elevating plasma glucose levels and stimulating further insulin secretion. Hepatic very low density lipoprotein (VLDL) export is subsequently enhanced, which leads to lipid deposition in extrahepatic tissues. Within pancreatic $\beta$ cells, excessive lipid deposition causes the metabolic inhibition of postprandial insulin secretion which, above an individual threshold, will herald the onset of hyperglycaemia. VLEDs are hypothesised to disrupt this flow of lipid between the liver and pancreas; within days of VLED commencement there is a rapid fall in IHCL and within weeks, reductions in pancreaticfat can be detected with a corresponding return in normal $\beta$-cell function.

Also noted during the early stages of weight-loss is a preferential loss of visceral adipose tissue (VAT) over SAT, thought to relate to its biological role as a short-term 
energy store.95 Findings from one systematic review suggest that, owing to the greater severity of ER, the percentage change in the VAT:SAT ratio is highest during rapid weight-loss associated with VLEDs, however this effect is thought to be transient. ${ }^{95}$ When compared to SAT, VAT depots typically possess higher levels of basal lipolysis, insensitivity to insulin action and macrophage infiltration, whilst adiponectin expression is reduced. ${ }^{3}$ As a result of the anatomical proximity and portal drainage of VAT to the liver, VATderived NEFA and pro-inflammatory adipokines are thought to act as a further driver for hepatic IR. ${ }^{3}$ Consequently, an intervention which is able to provide a sustained, preferential loss in VAT would be expected to provide significant metabolic and clinical benefits.

IER is effectively a regime consisting of short periods of very low (or no) energy intake alternated with habitual intake, rather than a continuous VLED. Previous reviews of the IER literature have speculated that repeated acute periods of negative energy balance experienced during IER may preferentially mobilize fat within visceral and intra-hepatic sites, perhaps more so than CER, owing to their acute sensitivities to ER. ${ }^{96,97}$ Corroborative evidence shall be discussed next.

Two studies, in rodents, have quantified changes in the distribution of VAT and SAT. One study in male mice following a fourweek IER (85\% ER/alternate days) showed that whilst total adipose-mass remained unchanged, there was a redistribution of adipose tissue from VAT to SAT, irrespective of high- or low-fat background diet. $^{53}$ In addition, this favourable shift in fat distribution corresponded to an elevation in plasma adiponectin levels. Subsequently, a direct comparison by Varady et $\mathrm{al}^{27}$, between CER (25\% ER/day) and three IER protocols (75\%; 85\% and $100 \%$ ER/alternate days), showed an equal efficacy of all four dietary regimes in promoting this redistribution and increase in adiponectin expression in female mice, despite only the CER group showing reduction in total fat-mass.
Studies in rodents have additionally described post-treatment elevations in the rate of fatty acid oxidation within both the liver and skeletal muscle of mice maintained on IER $(100 \%$ ER/alternate days). ${ }^{13}$ In a comparison of CER (40\% ER/day) and IER (100\% ER/alternate days), the authors of this 20-week study noted a doubling in the $\beta$-hydroxybutyrate levels (a marker of mitochondrial fatty acid oxidation) in IER-fed mice which was not present in the CER group. ${ }^{15}$ Both IER and CER interventions led to comparable reductions in fasting levels of glucose and insulin. Of particular interest however, is that total energy intake and body weights of IER-fed mice were comparable to ad libitum controls. This suggests that, unlike mice in the CER group, elevated fatty acid oxidation and improvements in glucose control of the IER-fed mice occurred in the absence of an overall energy deficit.

In humans, acute (measured after one to two ER-days) 33,37,48 and post-treatment 41, 48 increases in ketone bodies including $\beta$ hydroxybutyrate have been reported in individuals undergoing IER (75-100\% ER on restricted days). Also noted by two RCTs in overweight/obese women are acute reductions in HOMA-IR (reflective of hepatic insulin sensitivity) when measured after only two days of $75 \%$ ER, which may have resulted from the mobilization and subsequent oxidation of IHCL. ${ }^{41,} 48$ In both studies, insulin sensitivity, which was measured after five subsequent days of euenergetic intake, remained higher when compared to subjects assigned to a CER (25\% ER/day) protocol. On the other hand Soeters et al $^{34}$, who used a balanced crossover design, failed to note any effect of two weeks of IER (100\% ER/ alternate days) on hepatic insulin sensitivity (measured after an overnight fast) when compared to two weeks of a standard euenergetic diet. A particular strength of this study is its use of both isotopically labelled glucose and the hyperinsulinemic-euglycemic clamp technique, which is considered the gold standard method for assessment of insulin sensitivity, and allows for the differentiation between peripheral and hepatic insulin sensitivity. ${ }^{98}$ It should be noted however that this was a study of 
eight lean, glucose tolerant men and thus presents a possible limitation for these findings.

Studies utilizing stable isotope labelled tracers have also given an insight into the alterations in VLDL-kinetics which occur in response to acute ER. For instance, studies in healthy, lean women have shown that an acute negative energy balance induced by a one-day $3 \mathrm{MJ}$ energy-deficit can lower fasting VLDL-triglycerides by $-26 \%$ (relative to one day of euenergetic intake), which was subsequently attributed to a $21 \%$ reduction in hepatic VLDL section and $+12 \%$ increase in plasma clearance rate. ${ }^{99}$ On the other hand, a separate study by the authors failed to find any acute effects after a one-day 2MJ energy-deficit, suggesting greater degrees of ER are required to elicit acute alterations in VLDL-metabolism in the absence of weight-loss. ${ }^{100}$

Combining these lines of evidence, it could be suggested that recurrent periods of acute ER, through repeated stimulation of fatty acid oxidation, could have the potential to attenuate IHCL and VAT accumulation over a more chronic time scale. This presents a possible explanation for the apparent superiority of some IER regimens (versus CER) in improving fasting insulin sensitivity 41, 48, and for why metabolic improvements might occur in absence of overall ER. ${ }^{15}$ Given the largely indirect nature of the evidence however, direct quantification of changes in IHCL and VAT/SAT distribution in response to IER are needed. Use of stable isotope tracers would also be of particular value as they would allow for in vivo assessments of post-treatment alterations various aspects of hepatic metabolism including glucose metabolism, fat oxidation and VLDLkinetics which have been shown to be altered by acute periods of ER. ${ }^{101}$

Increased Stress Resistance: ER is associated with increased median and maximal life span across a variety of species and can confer protection against chronic diseases including T2DM and CVD. 102,103 One proposed mechanism thought to mediate these effects is an up-regulation of cellular stress resistance in response to ER, which may occur via a process called hormesis. Specifically, prolonged ER is thought to function as a long-term, lowintensity stressor, eliciting adaptive responses in cells and organisms, thus conferring resistance to oxidative, metabolic and genotoxic insults. ${ }^{104}$ Several hormetic response pathways have been identified involving enzymes (kinases and deacetylases including Sirtuins), transcription factor- regulation the expression of several major categories of proteins including chaperones, antioxidant enzymes and growth factors. 104

Accumulating data suggests that IER may also activate hormesis, and there has been some suggestion that it is the stress associated with fasting, or the alternating periods of anabolism and catabolism, rather than an overall ER is responsible. ${ }^{15}$, 105 IER (100\% ER / alternate days) in rodent studies has been shown to upregulate the expression of anti-oxidative 20 , 105 , anti-apoptotic 20,21 and pro-angiogenic ${ }^{21}$ factors. Also noted by IER studies are an increase in the expression levels of silent mating type information regulation 2 homolog 1 (SIRT1), an $\mathrm{NAD}^{+}$-dependent deacetylase. ${ }^{20}$ The expression of SIRT1, also increased by prolonged ER in rodents, is linked to the up-regulation of cellular stress resistance and improved outcomes in animal models of metabolic, neurodegenerative and inflammatory diseases. ${ }^{106,107}$ These findings have been suggestively accompanied by improvements in resilience to disease progression in rodent models of Type 1 diabetic nephropathy ${ }^{20}$, survival following induced ischaemic injury ${ }^{21}$ and a reduction in oxidative stress. ${ }^{105}$

In non-obese humans, an increase in SIRT1 expression within skeletal muscle has also been noted after two weeks of IER (100\% ER/alternate days). Stress resistance has not been assessed however and so the biological relevance of this finding is currently unknown. ${ }^{32}$ Several IER trials (75-85\% ER on restricted days) in overweight/obese populations have reported reductions in various markers of oxidative stress 37,41 , which in one study was accompanied by a complementary 
increase in the anti-oxidant uric acid. ${ }^{37}$ In a direct comparison of IER $75 \%$ ER for two days / week) and CER, both ER strategies displayed equal efficacy in reducing levels of fast-acting advanced oxidation protein products (AOPP) after six months, which displayed a tendency to occur earlier (i.e. at three months) in the IER group. ${ }^{41}$ Levels of slow-acting (i.e. long term) AOPP tended to decrease in the IER group and increase in the CER group which the authors proposed may have resulted from IER-induced activation of autophagy, a key homeostatic cellular process in which dysfunctional or unnecessary cellular proteins are degraded and recycled. ${ }^{41}$ On the other hand, a followup study using similar IER/CER protocols demonstrated comparable reductions in AOPP in both groups after three months. ${ }^{48}$

\section{Summary and Future Research Directions}

Current evidence from studies in rodents and humans demonstrates that IER (50$100 \%$ ER on restricted days) is capable of promoting weight-loss and/or favourably influencing an array of cardiometabolic health indices, with equal or greater efficacy than CER. In rodents, this has been shown to translate into improvements in clinical end-points such as disease progression. Putative mechanisms include the effects of IER on adipose physiology, stress resistance and acute ER-induced mobilization of fat distributed within visceral and intra-hepatic sites (Figure 1). However a large proportion of this mechanistic evidence is limited to indirect observations and/or has come from rodent studies requiring translation into humans. Furthermore, relatively few studies human studies (Table 1) have incorporated separate CER (standard treatment) or ad libitum (no intervention) control groups. Further investigations, using well designed RCTs, are clearly merited.

There is some indication that total IER and the array of partial IER protocols which have been developed, may elicit distinct biological effects to one another. The few rodent studies which have directly addressed this have demonstrated equivalence between total and the more severe partial IER strategies (50-100\% ER / alternate days) in modulating some aspects of adipose physiology. ${ }^{27,} 38,96 \mathrm{In}$ humans, the apparent superiority of twoday IER 41,48 , but not alternate-day IER 46 protocols over CER in improving fasting insulin sensitivity could be related to the greater length of the ER interval. Conversely, the extended fasting periods associated with total IER $(100 \%$ ER/alternate days) protocols have been linked to worsening post-prandial glucose control in women. ${ }^{32}$ It will thus be of interest to study and directly compare the metabolic effects of IER protocols which vary in frequency, duration and severity of ER periods.

Whilst initial findings are promising, the long-term efficacy, safety, and whether these metabolic improvements can be maintained during weight-stabilization, remain unknown. It will also be important to determine the effects of IER in other populations, for example in individuals with more profound metabolic derangements such as those with established T2DM, the evidence for which is relatively sparse. ${ }^{36}$ Additionally, given that several rodent and human IER (50$100 \%$ ER on restricted days) trials have demonstrated metabolic improvements in the absence of overall ER 15, 22, 27, 31, 53,105, one can speculate that IER may have potential applications within nonoverweight populations and as a maintenance strategy post-weight-loss.

Ultimately, while much remains to be known about IER, its modes of actions and long-term efficacy, the positive findings to date serve to highlight promising avenues for future research.

\section{References}

1. World Health Organization (WHO) (2013). "Obesity and Overweight: Factsheet Number 311," Available: http://www.who.int/mediacentre/fac tsheets/fs311/en/. Last accessed 11th March 2014.

2. Health and Social Care Information Centre (HSCIC 2013). "Health Survey 
for England 2012 - Adult Trend Tables," Available: http://www.hscic.gov.uk/catalogue/P UB13219. Last accessed 11th March 2014.

3. Leiter, L. A., Fitchett, D. H., Gilbert, R. E., Gupta, M., Mancini, G. B. J. et al. (2011). "Cardiometabolic Risk in Canada: A Detailed Analysis and Position Paper by the Cardiometabolic Risk Working Group," Canadian Journal of Cardiology, 27 (2), e1-e33.

4. World Health Organization (WHO) (2011). "NCD Country Profiles: United Kingdom," Available: http://www.who.int/nmh/countries/ gbr_en.pdf. Last accessed 15th Dec 2013.

5. Manco, M. \& Mingrone, G. (2005). "Effects of Weight Loss And Calorie Restriction On Carbohydrate Metabolism," Current Opinion In Clinical Nutrition And Metabolic Care., 8 (4), 431-9.

6. Henry, R. R., Scheaffer, L. \& Olefsky, J. M. (1985). "Glycemic Effects of Intensive Caloric Restriction and Isocaloric Refeeding in NoninsulinDependent Diabetes Mellitus," Journal of Clinical Endocrinology \& Metabolism, 61 (5), 917-925.

7. Kelley, D. E., Wing, R., Buonocore, C., Sturis, J., Polonsky, K. et al. (1993). "Relative Effects of Calorie Restriction and Weight Loss in NoninsulinDependent Diabetes Mellitus," Journal of Clinical Endocrinology Metabolism, 77 (5), 1287-93.

8. Taylor, R. (2013). "Banting Memorial Lecture 2012 Reversing the Twin Cycles of Type 2 Diabetes," Diabetic Medicine, 30 (3), 267-275.

9. Lim, E. L., Hollingsworth, K. G., Aribisala, B. S., Chen, M. J., Mathers, J. C. et al. (2011). "Reversal of Type 2 Diabetes: Normalisation of Beta Cell Function in Association with Decreased Pancreas and Liver
Triacylglycerol," Diabetologia, 54 (10), 2506-14.

10. Kolb, H. \& Eizirik, D. L. (2012). "Resistance to Type 2 Diabetes: A Matter of Hormesis?," Nature Reviews in Endocrinology, 8, 183-192.

11. Wing, R. R. \& Hill, J. O. (2001). "Successful Weight Loss Maintenance," Annual Review of Nutrition, 21 (1), 323-341.

12. Krízová, E. \& Simek, V. (1996a). "Influence of Intermittent Fasting and High-Fat Diet on Morphological Changes of the Digestive System and On Changes of Lipid Metabolism in the Laboratory Mouse," Physiological Research, 45 (2), 145-51.

13. Krízová, E. \& Simek, V. (1996b). "Effect of Intermittent Feeding with High-Fat Diet on Changes of Glycogen, Protein and Fat Content in Liver and Skeletal Muscle in the Laboratory Mouse," Physiological Research, 45 (5), 379-83

14. Pedersen, C. R., Hagemann, I., Bock, T. \& Buschard, K.. (1999). "Intermittent Feeding and Fasting Reduces Diabetes Incidence in BB Rats," Autoimmunity, 55 (30), 243-50.

15. Anson, R. M., Guo, Z., De Cabo, R., Iyun, T., Rios, M. et al. (2003). "Intermittent Fasting Dissociates Beneficial Effects of Dietary Restriction on Glucose Metabolism and Neuronal Resistance to Injury from Calorie Intake," Proceedings of the National Academy of Sciences, 100 (10), 6216-6220.

16. Wan, R., Camandola, S. \& Mattson, M. P. (2003a). "Intermittent Food Deprivation Improves Cardiovascular and Neuroendocrine Responses to Stress in Rats," The Journal of Nutrition, 133 (6), 1921-1929.

17. Wan, R., Camandola, S. \& Mattson, M. P. (2003b). "Intermittent Fasting and Dietary Supplementation with 2Deoxy-D-Glucose Improve Functional 
and Metabolic Cardiovascular Risk Factors in Rats," The FASEB Journal,17, 1133-1134.

18. Ahmet, I., Wan, R., Mattson, M. P., Lakatta, E. G. \& Talan, M. (2005). "Cardioprotection by Intermittent Fasting in Rats," Circulation, 112 (20) 3115-3121.

19. Mager, D. E., Wan, R., Brown, M., Cheng, A., Wareski, P. et al. (2006). "Caloric Restriction and Intermittent Fasting Alter Spectral Measures of Heart Rate and Blood Pressure Variability in Rats," The FASEB Journal, 20 (6), 631-637.

20. Tikoo, K., Tripathi, D. N., Kabra, D. G., Sharma, V. \& Gaikwad, A. B. (2007). "Intermittent Fasting Prevents the Progression of Type I Diabetic Nephropathy in Rats and Changes the Expression of Sir2 and p53," FEBS Letters, 581 (5), 1071-1078.

21. Katare, R. G., Kakinuma, Y., Arikawa, M., Yamasaki, F. \& Sato, T. (2009). "Chronic Intermittent Fasting Improves the Survival Following Large Myocardial Ischemia by Activation of BDNF/VEGF/PI3K Signaling Pathway," Journal of Molecular and Cellular Cardiology, 46 (3), 405-412.

22. Varady, K. A., Roohk, D. J., Loe, Y. C., McEvoy-Hein, B. K. \& Hellerstein, M. K. (2007b). "Effects of Modified Alternate-Day Fasting Regimens on Adipocyte Size, Triglyceride Metabolism, and Plasma Adiponectin Levels in Mice," Journal of Lipid Research, 48 (10), 2212-2219.

23. Ahmet, I., Wan, R., Mattson, M. P., Lakatta, E. G. \& Talan, M. I. (2010). "Chronic Alternate-Day Fasting Results in Reduced Diastolic Compliance and Diminished Systolic Reserve in Rats," Journal of Cardiac Failure, 16 (10), 843-853.

24. Castello, L., Froio, T., Maina, M., Cavallini, G., Biasi, F. et al. (2010).
"Alternate-Day Fasting Protects the Rat Heart Against Age-Induced Inflammation and Fibrosis by Inhibiting Oxidative Damage and NF$\mathrm{Kb}$ Activation," Free Radical Biology and Medicine, 48 (1), 47-54.

25. Razzak, R. L. A. \& Abu-Hozaifa, B. M. (2011). "Assessment of Enhanced Endothelium-Dependent Vasodilation by Intermittent Fasting in Wistar Albino Rats," Indian Journal Physiology Pharmacology, 55 (4), 336342.

26. Cerqueira, F. M., Da Cunha, F. M., Caldeira Da Silva, C. C., Chausse, B., Romano, R. L. et al. (2011). "LongTerm Intermittent Feeding, but Not Caloric Restriction, Leads to Redox Imbalance, Insulin Receptor Nitration, and Glucose Intolerance," Free Radical Biology and Medicine, 51 (7), 14541460.

27. Varady, K. A., Allister, C. A., Roohk, D. J. \& Hellerstein, M. K. (2010). "Improvements in Body Fat Distribution and Circulating Adiponectin by Alternate-Day Fasting Versus Calorie Restriction," The Journal of Nutritional Biochemistry, 21 (3), 188-195.

28. Jiang, H. Y., Koike, T., Li, P., Wang, Z. H., Kawata, Y. et al. (2010). "Combined Effects of Short-Term Calorie Restriction and Exercise on Insulin Action in Normal Rats," Hormone and Metabolic Research, 42 (13), 950-4.

29. Wan, R., Ahmet, I., Brown, M., Cheng, A., Kamimura, N. et al. (2010). "Cardioprotective Effect of Intermittent Fasting Is Associated with an Elevation of Adiponectin Levels in Rats," The Journal of Nutritional Biochemistry, 21 (5), 413417.

30. Castello, L., Maina, M., Testa, G., Cavallini, G., Biasi, F. et al. (2011). "Alternate-Day Fasting Reverses the Age-Associated Hypertrophy Phenotype in Rat Heart by Influencing the ERK and PI3K Signaling 
Pathways," Mechanisms of Ageing and Development, 132 (6-7), 305-314.

31. Halberg, N., Henriksen, M., Söderhamn, N., Stallknecht, B., Ploug, T. et al. (2005). "Effect of Intermittent Fasting and Refeeding on Insulin Action in Healthy Men," Journal of Applied Physiology, 99 (6), 2128-2136.

32. Heilbronn, L. K., Civitarese, A. E., Bogacka, I., Smith, S. R., Hulver, M. et al. (2005a). "Glucose Tolerance and Skeletal Muscle Gene Expression in Response to Alternate Day Fasting," Obesity Research, 13 (3), 574-581.

33. Heilbronn, L. K., Smith, S. R., Martin, C. K., Anton, S. D. \& Ravussin, E. (2005b). "Alternate-Day Fasting in Nonobese Subjects: Effects on Body Weight, Body Composition, and Energy Metabolism," The American Journal of Clinical Nutrition, 81 (1), 69-73.

34. Soeters, M. R., Lammers, N. M. Dubbelhuis, P. F., Ackermans, M., Jonkers-Schuitema, C. F. et al. (2009). "Intermittent Fasting Does Not Affect Whole-Body Glucose, Lipid or Protein Metabolism," American Journal Clinical Nutrition, 90 (5), 1244-1251.

35. Hill, J. O., Schlundt, D. G., Sbrocco, T., Sharp, T., Pope-Cordle, J. et al. (1989). "Evaluation of an Alternating-Calorie Diet with and Without Exercise in the Treatment of Obesity," The American Journal of Clinical Nutrition, 50 (2), 248-254.

36. Ash, S., Reeves, M. M., Yeo, S., Morrison, G., Carey, D. et al. (2003). "Effect of Intensive Dietetic Interventions on Weight and Glycaemic Control in Overweight Men with Type II Diabetes: A Randomised Trial," International Journal of Obesity, 27, 797-802.

37. Johnson, J. B., Summer, W. \& Mattson, M. P. (2007). "Alternate Day Calorie Restriction Improves Clinical Findings and Reduces Markers of Oxidative Stress and Inflammation in
Overweight Adults with Moderate Asthma," Free Radical Biology and Medicine, 42 (5), 665-674.

38. Varady, K. A., Bhutani, S., Church, E. C. \& Klempel, M. C. (2009a). "Short-Term Modified Alternate-Day Fasting: A Novel Dietary Strategy for Weight Loss and Cardioprotection in Obese Adults," The American Journal of Clinical Nutrition, 90 (5), 1138-1143.

39. Bhutani, S., Klempel, M. C., Berger, R. A. \& Varady, K. A. (2010). "Improvements in Coronary Heart Disease Risk Indicators by AlternateDay Fasting Involve Adipose Tissue Modulations," Obesity, 18 (11), 21522159.

40. Eshghinia, S. \& Gapparov, M. G. (2011). "Effect of Short-Term Modified Alternate-Day Fasting on the Lipid Metabolism in Obese Women," Iranian Journal of Diabetes and Obesity, 3 (1), 1-5.

41. Harvie, M. N., Pegington, M., Mattson, M. P., Frystyk, J., Dillon, B. et al. (2011). "The Effects of Intermittent or Continuous Energy Restriction on Weight Loss and Metabolic Disease Risk Markers: A Randomised Trial in Young Overweight Women," International Journal of Obesity, 35, 714-727.

42. Varady, K. A., Bhutani, S., Klempel, M. C. \& Kroeger, C. M. (2011a). "Comparison of Effects of Diet versus Exercise Weight Loss Regimens on LDL and HDL Particle Size in Obese Adults," Lipids in Health and Disease, $10,119$.

43. Varady, K. A., Bhutania, S., Klempel, M. C. \& Lamarchea, B. (2011b). "Improvements in LDL Particle Size and Distribution by Short-Term Alternate Day Modified Fasting in Obese Adults," British Journal of Nutrition, 105, 580-583.

44. Klempel, M. C., Kroeger, C. M., Bhutani, S., Trepanowski, J. F. \& Varady, K. A. 
(2012). "Intermittent Fasting Combined with Calorie Restriction Is Effective for Weight Loss and CardioProtection in Obese Women," Nutrition Journal, 11 (98), http://www.nutritionj.com/content/ 11/1/98.

45. Eshghinia, S. \& Mohammadzadeh, F. (2013) "The Effects of Modified Alternate-Day Fasting Diet on Weight Loss and CAD Risk Factors in Overweight and Obese Women," Journal of Diabetes \& Metabolic Disorders, $12 \quad$ (4), http://www.jdmdonline.com/content $/ 12 / 1 / 4$.

46. Bhutani, S., Klempel, M. C., Kroeger, C. M., Trepanowski, J. F. \& Varady, K. A. (2013a). "Alternate Day Fasting and Endurance Exercise Combine to Reduce Body Weight and Favorably Alter Plasma Lipids in Obese Humans," Obesity, 21 (7), 1370-1379.

47. Bhutani, S., Klempel, M. C., Kroeger, C. M., Trepanowski, J. F., Phillips, S. A. et al. (2013b). "Alternate Day Fasting with or without Exercise: Effects on Endothelial Function and Adipokines in Obese Humans," e-SPEN Journal, 8 (5), E205-E209.

48. Harvie, M., Wright, C., Pegington, M., Mcmullan, D., Mitchell, E. et al. (2013). "The Effect of Intermittent Energy and Carbohydrate Restriction V. Daily Energy Restriction on Weight Loss and Metabolic Disease Risk Markers in Overweight Women," British Journal of Nutrition, 110 (08), 15341547.

49. Klempel, M. C., Kroeger, C. M., Norkeviciute, E., Goslawski, M., Phillips, S. A. et al. (2013a). "Benefit of a Low-Fat Over High-Fat Diet on Vascular Health during Alternate Day Fasting," Nutr Diabetes, 3 (5), E71.

50. Klempel, M. C., Kroeger, C. M. \& Varady, K. A. (2013b). "Alternate Day Fasting Increases LDL Particle Size Independently of Dietary Fat Content in Obese Humans," European Journal of Clinical Nutrition, 67, 783-785.

51. Klempel, M. C., Kroeger, C. M. \& Varady, K. A. (2013c). "Alternate Day Fasting (ADF) with a High-Fat Diet Produces Similar Weight Loss and Cardio-Protection as ADF with a LowFat Diet," Metabolism, 62 (1), 137-143.

52. Varady, K. A., Bhutani, S., Klempel, M. C. Kroeger, C. M., Trepanowski, J. F. Haus, J. M., Hoddy, K. K. \& Calvo, Y. (2013). "Alternate Day Fasting for Weight Loss in Normal Weight and Overweight Subjects: A Randomized Controlled Trial," Nutrition Journal, 12, 146.

53. Varady, K. A., Hudak, C. S. \& Hellerstein, M. K. (2009b). "Modified Alternate-Day Fasting and Cardioprotection: Relation to Adipose Tissue Dynamics and Dietary Fat Intake," Metabolism, 58 (6), 803-811.

54. Higashida, K., Fujimoto, E., Higuchi, M. \& Terada, S. (2013). "Effects of Alternate-Day Fasting on High-Fat Diet-Induced Insulin Resistance in Rat Skeletal Muscle," Life Sciences, 93 (56), Pp. 208-213.

55. Henriksen, E. J., Bourey, R. R., Rodnick, K. J., Koranyi, M. A. Permutt, J. O. et al. (1990). "Glucose Transporter Protein Content and Glucose Transport Capacity in Rat Skeletal Muscles," American Journal Physiology Endocrinology Metabolism, 259 (1990), E593-598.

56. Robertson, M. D., Livesey, G. \& Mathers, J. C. (2002). "Quantitative Kinetics of Glucose Appearance and Disposal Following a 13C-Labelled Starch-Rich Meal: Comparison of Male and Female Subjects," British Journal of Nutrition, 87 (6), 569-77.

57. Soeters, M. R., Soeters, P. B., Schooneman, M. G., Houten, S. M. \& Romijn, J. A. (2012). "Adaptive Reciprocity of Lipid and Glucose Metabolism in Human Short-Term 
Starvation," American Journal of Physiology - Endocrinology and Metabolism, 303, E1397-E1407

58. Horton, T. J. \& Hill, J. O. (2001). "Prolonged Fasting Significantly Changes Nutrient Oxidation and Glucose Tolerance after a Normal Mixed Meal," Journal of Applied Physiology, 90 (1), 155-163

59. Féry, F., d'Attellis, N. P. \& Balasse, E. O. (1990). "Mechanisms of Starvation Diabetes: A Study with Double Tracer and Indirect Calorimetry," American Journal of Physiology, 259, E770E777.

60. Node, K. \& Inaeu, T. (2009). "Postprandial Hyperglycaemia as an Eitiological Risk Factor in Vascular Failure," Cadiovascular Diabetology, 8, 23.

61. Kumar, P. \& Clark, M. L. (2009). Clinical Medicine 7th Revised Edition, Saunders Ltd, London, United Kingdom.

62. Goodpaster, B. H., Kelley, D. E., Wing, R. R., Meier, A. \& Thaete, F. L. (1999). "Effects of Weight Loss on Regional Fat Distribution and Insulin Sensitivity in Obesity," Diabetes, 48 (4), 839-847.

63. Ross, R., Dagnone, D., Jones, P. J. et al. (2000). "Reduction in Obesity and Related Comorbid Conditions after Diet-Induced Weight Loss or ExerciseInduced Weight Loss in Men: A Randomized, Controlled Trial," Annals of Internal Medicine, 133, 92-103.

64. Cox, K. L., Burke, V., Morton, A. R., Beilin, L. J. \& Puddey, I. B. (2004). "Independent and Additive Effects of Energy Restriction and Exercise on Glucose and Insulin Concentrations in Sedentary Overweight Men," The American Journal of Clinical Nutrition, 80 (2), 308-316.

65. Ross, R., Janssen, I., Dawson, J., Kungl, A. M., Kuk, J. L. et al. (2004). "ExerciseInduced Reduction in Obesity and
Insulin Resistance in Women: A Randomized Controlled Trial," Obesity Research, 12, 789-98.

66. Kasuga, M. (2006). "Insulin Resistance and Pancreatic $\beta$ Cell Failure," Journal of Clinical Investigation, 116 (7), 1756-1760.

67. Tabak, A. G., Jokela, M., Akbaraly, T. N., Brunner, E. J., Kivimaki, M. \& Witte, D. R. (2009). "Trajectories of Glycaemia, Insulin Sensitivity, and Insulin Secretion before Diagnosis of Type 2 Diabetes: An Analysis from the Whitehall II Study," The Lancet; 373: 2215-2221.

68. Warram, J. H., Martin, B. C., Krolewski, A. S., Soeldner, J. S. \& Kahn, C. R. (1990). "Slow Glucose Removal Rate and Hyperinsulinemia Precede the Development of Type 2 Diabetes in the Offspring of Diabetic Parents," Annals of Internal Medicine, 113, 909915.

69. Martin, B. C., Warram, J. H., Krolewski, A. S., Bergman, R. N., Soeldner, J. S. \& Kahn, C. R. (1992). "Role of Glucose and Insulin Resistance in Development of Type 2 Diabetes Mellitus: Results of a 25-Year FollowUp Study," The Lancet, 340 (8825), 925-9.

70. Weyer, C., Hanson, R. L., Tataranni, P. A., Bogardus, C. \& Pratley, R. E. (2000). "A High Fasting Plasma Insulin Concentration Predicts Type 2 Diabetes Independent of Insulin Resistance: Evidence for a Pathogenic Role of Relative Hyperinsulinemia," Diabetes, 49 (12), 2094-2101.

71. Matthews, D. R., Hosker, P., Rudenski, A. S. et al. (1985). "Homeostasis Model Assessment: Insulin Resistance and Beta-Cell Function from Fasting Plasma Glucose and Insulin Concentrations in Man," Diabetologia, 28, 412-419.

72. Mattson, M. P. \& Wan, R. (2005). "Beneficial Effects of Intermittent 
Fasting and Caloric Restriction on the Cardiovascular and Cerebrovascular Systems," The Journal of Nutritional Biochemistry, 16 (3), 129-137.

73. Nordestgaard, B. G. \& Zilversmit, D. B. (1989). "Comparison of Arterial Intimal Clearances of LDL from Diabetic and Nondiabetic CholesterolFed Rabbits. Differences in Intimal Clearance Explained by Size Differences," Arteriosclerosis, 9, 17683.

74. De Graaf, J., Hak-Lemmers, H. L., Hectors, M. P., Demacker, P. N., Hendriks, J. C. et al. (1991). "Enhanced Susceptibility to in Vitro Oxidation of the Dense Low Density Lipoprotein Subfraction in Healthy Subjects," Arteriosclerosis, Thrombosis, and Vascular Biology, 11 (2), 298-306.

75. Tribble, D. L., Holl, L. G., Wood P. D. \& Krauss, R. M. (1999). "Variations in Oxidative Susceptibility among Six Low Density Lipoprotein Subfractions of Differing Density and Particle Size," Atherosclerosis, 93, Pp. 189-99

76. Kroeger, C. M., Klempel, M. C., Bhutani, S., Trepanowski, J. F., Tangney, C. C. et al. (2012). "Improvement in Coronary Heart Disease Risk Factors during an Intermittent Fasting/Calorie Restriction Regimen: Relationship to Adipokine Modulations," Nutrition \& Metabolism, 9 (98), http://www.nutritionandmetabolism. com/content/9/1/98.

77. Barter, P. (2005). "The Role of HDLCholesterol in Preventing Atherosclerotic Disease," European Heart Journal Supplements, 7 (Suppl F), F4-F8.

78. Dattilo, A. M. \& Kris-Etherton, P. M. (1992). "Effects of Weight Reduction on Blood Lipids and Lipoproteins: A Meta-Analysis," The American Journal of Clinical Nutrition, 56 (2), 320-328.

79. Vanhoutte, P. M., Shimokawa, H., Tang, E. H. C. \& Feletou, M. (2009).
"Endothelial Dysfunction and Vascular Disease," Acta Physiologica, 196 (2), 193-222.

80. Van Der Meer, R. W., Hammer, S., Smit, J. W. M., Frölich, M., Bax, J. J. et al. (2007). "Short-Term Caloric Restriction Induces Accumulation of Myocardial Triglycerides and Decreases Left Ventricular Diastolic Function in Healthy Subjects," Diabetes, 56, 2849-2853.

81. Widya, R. L., Hammer, S., Boon, M. R., Van Der Meer, R. W., Smit, J. W. A. et al. "Effects of Short-Term Nutritional Interventions on Right Ventricular Function in Healthy Men," PloS One, 8 (9), E76406.

82. Maclean, P. S., Bergouignan, A., Cornier, M. A. \& Jackman, M. R. (2011). "Biology's Response to Dieting: The Impetus for Weight Regain," American Journal of Physiology Regulatory Integrative and Comparative Physiology, 301 (3), R581-600.

83. Rosenbaum, M., Sy, M., Pavlovich, K., Leibel, R. L. \& Hirsch, J. (2008). "Leptin Reverses Weight-Loss Induced Changes in Regional Neural Activity Responses to Visual Food Stimuli," Journal of Clinical Investigation, 118 (7): 2583-2591.

84. Klempel, M. C., Bhutani, S., Fitzgibbon, M., Freels, S. \& Varady, K. A. (2010). "Dietary and Physical Activity Adaptations to Alternate Day Modified Fasting: Implications for Optimal Weight Loss," Nutrition Journal, 9 (35), http://www.nutritionj.com/content/ 9/1/35.

85. Flint, A., Raben, A., Blundell, J. E. \& Astrup, A. (2000). "Reproducibility, Power and Validity of Visual Analogue Scales in Assessment of Appetite Sensations in Single Test Meal Studies," International Journal of Obesity and Related Metabolic Disorders, 24(1), 38-48. 
86. Paddon-Jones, D., Westman, E., Mattes, R. D. et al. (2008). "Protein, Weight Management, and Satiety," American Journal of Clinical Nutrition, 87 (5), 15585-15615.

87. Klok, M. D. Jakobsdottir, S. \& Drent, M. L. (2006). "The Role of Leptin and Ghrelin in the Regulation of Food Intake and Body Weight in Humans: A Review," Obesity Reviews, 8 (1), 21-34.

88. Cummings, D. E., Weigle, D. S., Frayo, R. S. et al. (2002). "Plasma Ghrelin Levels after Diet-Induced Weight Loss or Gastric Bypass Surgery," New England Journal of Medicine, 346, 1623-30.

89. English, P. J., Ghatei, M. A., Malik, I. A., Bloom, S. R. \& Wilding, J. P. H. (2002). "Food Fails to Suppress Ghrelin Levels in Obese Humans," Journal of Clinical Endocrinology Metabolism, 87, 2984.

90. Frayn, K. N. (2002). "Adipose Tissue as a Buffer for Daily Lipid Flux," Diabetologia, 45 (9), 1201-10.

91. Hajer, G. R., Van Haeften, T. W. \& Visseren, F. L. J. (2008). "Adipose Tissue Dysfunction in Obesity, Diabetes, and Vascular Diseases," European Heart Journal, 29 (24), Pp. 2959-2971.

92. Bays, H. E. (2011). "Adiposopathy Is "Sick Fat" A Cardiovascular Disease?," Journal of the American College of Cardiology, 57, 2461-2473.

93. Després, J., Lemieux, I., Bergeron, J., Pibarot, P., Mathieu, P. et al. (2008). "Abdominal Obesity and the Metabolic Syndrome: Contribution to Global Cardiometabolic Risk," Arteriosclerosis, Thrombosis, and Vascular Biology, 28 (6), 1039-1049.

94. Eklund, C. M. (2009). "Proinflammatory Cytokines in CRP Baseline Regulation," Advanced Clinical Chemistry, 48, 111-36.
95. Chaston, T. B. \& Dixon, J. B. (2008). "Factors Associated with Percent Change in Visceral Versus Subcutaneous Abdominal Fat during Weight Loss: Findings from a Systematic Review," International Journal of Obesity, 32, 619-628.

96. Varady, K. A. \& Hellersteine, M. K. (2007a). "Alternate-Day Fasting and Chronic Disease Prevention: A Review of Human and Animal Trials," American Journal of Clinical Nutrition, 86 (1), 7-13.

97. Harvie, M. \& Howell, A. (2012). "Energy Restriction and the Prevention of Breast Cancer," Proceedings of the Nutrition Society, 71 (2), 263-275.

98. Kim, J. K. (2009). "HyperinsulinemicEuglycemic Clamp to Assess Insulin Sensitivity in Vivo," Methods Molecular Biology, 560, 221-238.

99. Bellou, E., Maraki, M., Magkos, F., Botonaki, H., Panagiotakos, D. B. et al. (2013a). "Effect of Acute Negative and Positive Energy Balance on Basal Very-Low Density Lipoprotein Triglyceride Metabolism in Women," PloS One, 8 (3), E60251.

100. Bellou, E., Siopi, A., Galani, M., Maraki, M., Tsekouras, Y. E. et al. (2013b). "Acute Effects of Exercise and Calorie Restriction on Triglyceride Metabolism in Women," Medicine and Science in Sports Exercise, 45, 455461.

101. Magkos, F. \& Mittendorfer, B. (2009). "Stable Isotope-Labeled Tracers for the Investigation of Fatty Acid and Triglyceride Metabolism in Humans in Vivo," Clinical Lipidology, 4 (2), 215230.

102. Mcdonald, R. B. \& Ramsey, J. J. (2010). "Honoring Clive McCay and 75 Years of Calorie Restriction Research," Journal of Nutrition,140 (7), 12051210. 
103. Bordone, L. \& Guarente, L. (2005). "Calorie Restriction, SIRT1 and Metabolism: Understanding Longevity," Nature Reviews Molecular Cell Biology, 6, 298-305.

104. Mattson, M. P. (2008). "Hormesis Defined," Ageing Research Reviews, 7 (1), 1-7.

105. Descamps, O., Riondel, J., Ducros, V. \& Roussel, A. (2005). "Mitochondrial Production of Reactive Oxygen Species and Incidence of AgeAssociated Lymphoma in OF1 Mice: Effect of Alternate-Day Fasting," Mechanisms of Ageing and Development, 126 (11), 1185-1191.
106. Cohen, H. Y., Miller, C., Bitterman, K. J., Wall, N. R., Hekking, B., Kessler, B., Howitz, K. T., Gorospe, M., De Cabo, R. \& Sinclair, D. A. (2004). "Calorie Restriction Promotes Mammalian Cell Survival by Inducing the SIRT1 Deacetylase," Science, 305, 390-392.

107. Lavu, S., Boss, O., Elliott, P. J. \& Lambert, P. D. (2008). "Sirtuins Novel Therapeutic Targets to Treat Age-Associated Diseases," Nature Reviews, 7, 841-853. 